\title{
Aplicação das técnicas não- destrutivas de ultra-som, vibração transversal e ondas de tensão para avaliação de madeira
}

\author{
Application of nondestructive ultrasound techniques , \\ transverse vibration and stress-wave for timber \\ evaluation
}

\section{Marcela Candian Almir Sales}

Resumo

plicação de técnicas não-destrutivas pode auxiliar no processo de avaliação da madeira, não sendo necessária a extração de corpos-deprova. Entre as técnicas de avaliação mecânica, destacam-se as técnicas não-destrutivas de ultra-som e de vibração transversal. Com o avanço no uso das técnicas não-destrutivas, tornou-se possível o desenvolvimento de novas tecnologias, entre as quais se destaca a técnica de ondas de tensão, que permite a obtenção de imagem tomográfica da madeira. O objetivo deste trabalho foi verificar a acuracidade das técnicas de vibração transversal e ultra-som comparativamente aos valores obtidos em ensaios estáticos e a obtenção de imagem tomográfica a partir do emprego de ondas de tensão para a espécie Eucalyptus citriodora. Os valores obtidos de coeficiente de determinação para as técnicas de ultra-som $\left(R^{2}=0,76\right)$ e vibração transversal $\left(R^{2}=0,80\right)$ são significativos, demonstrando que essas são importantes ferramentas para a inferência do módulo de elasticidade da madeira. Os resultados permitem também verificar que a imagem tomográfica obtida com o emprego de ondas de tensão possibilita estimar a porcentagem da área deteriorada com adequada confiabilidade.

Palavras-chave: Ensaio não-destrutivo. Ultra-som. Vibração transversal. Ondas de tensão.

Marcela Candian Programa de Pós-Graduação, em Engenharia Urbana, Departamento de Engenharia

Universidade Federal de São Carlos Rodovia Washington Luiz, Km235 São Carlos - SP - Brasil Caixa-Postal: 676 CEP 13565-905

Tel.: (16) 3351-9659

marcela_candian@yahoo.com.br

Almir Sales

Departamento de Engenharia Civil

Universidade Federal de São Carlos

E-mail: almir@pq.cnpq.br

Recebido em 12/07/09

Aceito em 19/10/09

\section{Abstract}

The application of nondestructive techniques can assist in timber evaluation, without the need to extract specimens. Two of the main mechanic evaluation techniques are the nondestructive techniques of ultrasound and transversal vibration. The evolution in the use of nondestructive techniques has led to the development of new technologies, particularly the stress-wave technique, which allows the obtention of tomographic images of timber. The purpose of this study was to verify the accuracy of the ultrasonic and transverse vibration techniques in comparison with the values obtained from static tests and tomographic images obtained through the use of stress waves for Eucalyptus citriodora. The values of the coefficient of determination for the ultrasonic technique $\left(R^{2}=0.76\right)$ and for the transverse vibration technique $\left(R^{2}=0.80\right)$ were significant, demonstrating that the ultrasonic and transverse vibration techniques are important tools for the nondestructive inference of the modulus of elasticity of timber. The results also show that the tomographic images obtained through the use of the stress-wave technique allow the percentage of deteriorated area of timber to be estimated with adequate reliability.

Keywords: Nondestructive testing. Ultrasonic. Transverse vibration. Stress wave. 


\section{Introdução}

A madeira é um material orgânico, de origem vegetal, uma matéria-prima inesgotável, pois se encontra em contínua formação sob a forma de árvores, em florestas naturais ou florestas plantadas. Por ser um material natural, apresenta uma grande variabilidade em suas propriedades mecânicas. Devido a essa variabilidade, seu aproveitamento adequado pode ser alcançado com a classificação de todas as peças de madeira.

A madeira tem suas propriedades, em grande parte das situações, avaliadas pelo emprego de métodos destrutivos, que utilizam corpos-de-prova para a avaliação. Uma amostragem incorreta pode acarretar resultados não coerentes, e em alguns casos as propriedades do corpo-de-prova podem não ser representativas de um lote de madeira.

Uma avaliação adequada pode ser auxiliada pelo emprego de métodos não-destrutivos, pois estes permitem avaliar o próprio elemento, sem a necessidade de extração de corpos-de-prova. A avaliação não-destrutiva é definida como sendo a ciência de identificar as propriedades físicas e mecânicas de uma peça de determinado material sem alterar suas capacidades de uso final.

Os ensaios não-destrutivos são aqueles realizados em materiais para verificar a existência, ou não, de descontinuidades ou defeitos, por meio de princípios físicos definidos, sem alterar suas características físicas, químicas, mecânicas ou dimensionais, e sem interferir em seu uso posterior (ASSOCIAÇÃO BRASILEIRA DE ENSAIOS NÃO-DESTRUTIVOS E INSPEÇÃO, 2006).

Em materiais homogêneos e isotrópicos, a avaliação não-destrutiva permite detectar falhas surgidas em processos de fabricação. Na madeira, essas irregularidades ocorrem naturalmente, pois se trata de um material gerado pela natureza. A influência dessas irregularidades da madeira sobre as propriedades mecânicas pode ser avaliada de forma não-destrutiva.

Existem atualmente diversas técnicas de avaliação não-destrutiva que são empregadas para a avaliação da madeira, entre as quais se pode destacar: ultra-som, vibração transversal e ondas de tensão.

O ultra-som é uma técnica não-destrutiva baseada em propagação de ondas que apresenta muitas aplicações, tanto em árvores quanto em peças estruturais. A determinação das propriedades mecânicas da madeira usando propagação de ondas ultra-sônicas é baseada na relação entre a velocidade do som, o módulo de elasticidade e a densidade.
A técnica não-destrutiva por meio de ultra-som apresenta diversas finalidades, permitindo desde a determinação da existência de nós, presença de ataque de microorganismos ou insetos, direcionamento das fibras, decomposição, passando pela avaliação de elementos estruturais de madeira em uso, até a estimativa de alguns parâmetros como o módulo de elasticidade (GORNIAK; MATOS, 2000).

A utilização do ultra-som na engenharia civil iniciou-se na década de 1950, na Europa, sendo empregado inicialmente em investigações de concreto. Posteriormente, iniciaram-se estudos teóricos para a aplicação do ultra-som na madeira, sendo encontradas algumas dificuldades devido às peculiaridades anatômicas do material.

Os primeiros resultados experimentais foram obtidos por Waubke, em 1983, na Alemanha (SANDOZ, 1989). Atualmente, diversas pesquisas têm sido desenvolvidas para comprovar a validade da técnica de ultra-som na classificação de peças de madeira.

A técnica de ultra-som apresenta diversas vantagens, tais como baixo custo de aquisição do equipamento, quando comparado ao custo de máquinas de classificação automática, facilidade de treinamento da mão-de-obra e utilização pelas indústrias para melhorar o controle de qualidade dos processos por meio de uma maior uniformidade na matéria-prima e em seus derivados

A avaliação não-destrutiva com o emprego de vibração transversal é baseada na relação existente entre a rigidez e a freqüência natural de vibração (CARREIRA et al., 2004).

A técnica de vibração transversal foi um dos primeiros métodos não-destrutivos investigados para a determinação do módulo de elasticidade da madeira. Apesar de sua concepção simples, e a despeito dos grandes avanços obtidos nessa área com outros métodos, o método revela-se como de grande potencial de aplicação, sobretudo pela precisão do modelo matemático a ele associado e pela possibilidade de sua aplicação em peças de dimensões estruturais (in-grade testing) (BALLARIN; TARGA; PALMA, 2002).

O primeiro ensaio empregando a técnica de vibração transversal foi realizado por um cientista francês, para a determinação do módulo de elasticidade de uma barra de ferro. Posteriormente, esses resultados foram comparados com resultados obtidos em ensaios de tração em barras de ferro, sendo uma das primeiras tentativas de comparar 
valores de constantes elásticas obtidos em ensaios dinâmicos com valores resultantes de ensaios estáticos.

Com o passar dos anos, novas pesquisas foram desenvolvidas empregando-se a técnica de vibração transversal, o que comprovou a possibilidade do uso de características vibracionais para a estimativa do módulo de elasticidade estático. A técnica de vibração transversal é considerada atualmente um teste que apresenta valores com alta confiabilidade para o módulo de elasticidade da madeira (MURPHY, 2000).

A madeira, além da variabilidade própria de suas propriedades mecânicas, pode, ao longo dos anos, sofrer ação de diversos agentes degradadores, que causam danos severos ao material e a seus produtos (FURTADO, 2000).

A avaliação da deterioração, principalmente a degradação que não apresenta indicadores externos, é uma importante ferramenta para a inspeção de peças de madeira. Com o avanço no uso das técnicas não-destrutivas, foi possível o desenvolvimento de tecnologias para a obtenção de imagem tomográfica da madeira, que permite a localização de defeitos internos. A imagem nãodestrutiva é baseada em diferentes métodos que utilizam ondas elásticas, radiação, luz, campos magnéticos, elétricos e raios X (BUCUR, 2003; EVANS; ILIC, 2001; EVANS; KIBBLEWHITE, 2000; HAUFFE; MAHLER, 2000; NICOLOTTI et al., 2003; QUOIRIN, 2004; RUST, 2000). A imagem pode ser reconstruída através do mapeamento de diversos parâmetros e com o uso de procedimentos computacionais para o processamento dos resultados, reconstrução e exibição da imagem.

Pesquisas internacionais com várias técnicas baseadas em diferentes conceitos, como, por exemplo, raios $\mathrm{X}$, ressonância magnética e ondas de tensão, têm sido realizadas para a avaliação de peças de madeira, as quais fornecem imagens que possibilitam a localização de defeitos e a avaliação das características internas do material, mas apresentam uma limitação a seu uso devido ao alto custo de utilização. A técnica de ondas de tensão tem como vantagem o custo menor para sua implantação. Trata-se de um processo dinâmico que está internamente relacionado com as propriedades físicas e mecânicas da madeira.

A propagação de ondas de tensão apresenta como principal vantagem a possibilidade do estudo do interior da madeira, preservando as características físicas e mecânicas e seu uso final. $\mathrm{O}$ equipamento de ondas de tensão é capaz de detectar de forma não-destrutiva a presença de furos, deterioração e rachaduras.
No método de ondas de tensão, é analisada a velocidade de propagação de uma onda de tensão induzida e sua atenuação no material. Para explicar o método, é possível fazer uso da teoria de propagação de ondas unidirecionais em uma barra homogênea e elástica.

Ao aplicar um impacto em uma de suas extremidades, gera-se uma onda que se movimenta ao longo da barra, excitando as partículas da extremidade que recebeu o impacto, enquanto as partículas da extremidade livre se encontram em repouso. A onda se propaga a uma velocidade constante ao longo da barra e, logo após caminhar por todo o comprimento, é refletida pela extremidade livre da barra, retornando a seu ponto de origem.

Apesar de a velocidade continuar sempre constante, o movimento das partículas da barra vai diminuindo a cada passagem da onda pela barra. Após algum tempo, as partículas da barra retornam ao repouso. Verifica-se próximo da extremidade excitada uma série de pulsos igualmente espaçados, com a amplitude diminuindo ao longo do tempo.

A propagação de ondas na madeira é um processo dinâmico que está internamente relacionado com as propriedades físicas e mecânicas do material. As ondas longitudinais são as mais velozes e mais comumente usadas para avaliar as propriedades da madeira.

Este trabalho, ao realizar um estudo experimental sobre a avaliação da madeira com emprego de técnicas não-destrutivas, busca validar o emprego de técnicas não-destrutivas de ultra-som, vibração transversal e ondas de tensão para a avaliação da madeira, tanto na determinação de suas propriedades mecânicas, quanto na localização de defeitos internos, permitindo a racionalização do uso do material e a confiabilidade necessária para seu emprego na engenharia, de forma que a madeira possa ser empregada com a mesma confiabilidade com que são empregados outros materiais como o aço e o concreto.

\section{Materiais e métodos}

Neste trabalho foram empregadas 30 peças com dimensões estruturais de $5 \mathrm{~cm} \times 11 \mathrm{~cm} \times 300 \mathrm{~cm}$ da espécie Eucalyptus citriodora, as quais foram avaliadas utilizando-se as técnicas de ultra-som, vibração transversal e flexão estática. Para a obtenção da imagem tomográfica, foi empregado um disco de madeira da espécie Eucalyptus citriodora com $10 \mathrm{~cm}$ de espessura e $35 \mathrm{~cm}$ de diâmetro. $\mathrm{O}$ teor de umidade encontrado nas peças estruturais e no disco de madeira foi de $12 \%$, não 
sendo necessário corrigir as propriedades mecânicas em função do teor de umidade.

A aplicação da técnica de ultra-som foi realizada utilizando-se o equipamento Sylvatest com transdutores de $22 \mathrm{kHz}$, o qual pode ser visualizado na Figura 1. O equipamento possui entrada e saída de dados que possibilitam o armazenamento deles por meio da utilização de um notebook. É equipado com dois transdutores piezoeletrônicos, um gerando a onda (transmissor) e o outro recebendo-a (receptor). Ambos trabalham diretamente em contato com a madeira. O equipamento permite registrar o tempo de passagem da onda, em microssegundos, o que possibilita o cálculo do módulo de elasticidade dinâmico.

No momento em que se faz uso de métodos estatísticos para avaliação de experimentos, ressalta-se a importância de um planejamento prévio para que se obtenha, a um custo mínimo, a maior quantidade de informações sobre as características dos fatores considerados. Quando, por exemplo, não é determinado previamente o tamanho ótimo de uma amostra, pode-se utilizar um número mais elevado de repetições para tirar as mesmas conclusões que poderiam ser obtidas a partir de uma amostra de menor tamanho. Por outro lado, um número insuficiente de amostras pode gerar incertezas que impossibilitem qualquer conclusão referente aos fatores estudados. Além disso, ensaios não sistematizados, quando muitos fatores estão envolvidos, dificultam ou mesmo impedem qualquer análise estatística posterior.

$\mathrm{O}$ equipamento foi posicionado nas peças (Figura 2), sendo realizadas três leituras de tempo em microssegundos, com a onda se propagando na direção longitudinal às fibras, que foram medidas em três pontos distintos da seção transversal da peça, a partir das quais se determinou a média. Com o tempo médio, foi determinada a velocidade, e a partir desta, utilizando-se a eq. 1, foi possível determinar o módulo de elasticidade dinâmico (Ed, us).

$\mathrm{Ed}, \mathrm{us}=\rho \mathrm{x} \mathrm{v} 2$

onde:

Ed,us é o módulo de elasticidade dinâmico (10-6 $\mathrm{MPa}$ );

$\rho$ é a densidade da madeira $\left(\mathrm{kg} / \mathrm{m}^{3}\right)$; e

v é a velocidade da onda longitudinal (m/s).

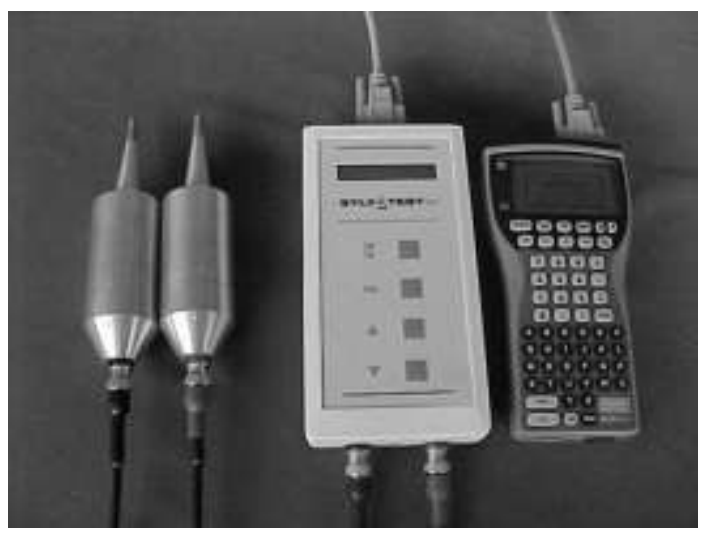

Figura 1 - Equipamento de ultra-som

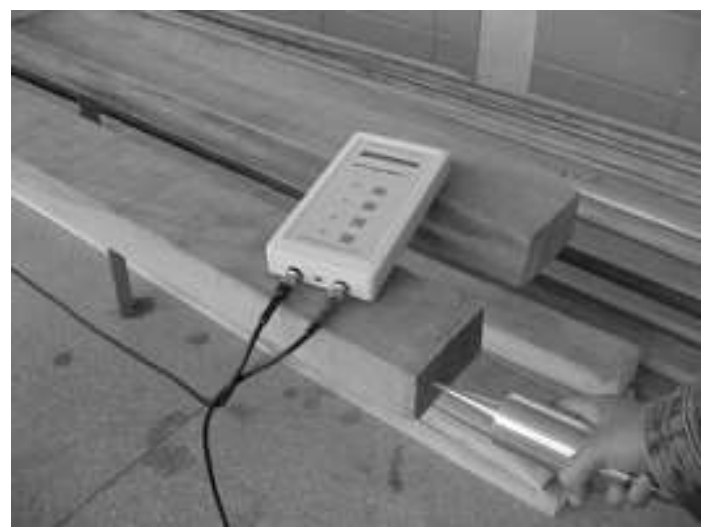

Figura 2 - Ensaio de ultra-som 
Para a aplicação da técnica de vibração transversal, foi empregado o equipamento Transverse Vibration E-Computer, da Metriguard. As peças de madeira foram dispostas biapoiadas, e aplicou-se um impacto no centro da peça (Figura 3). A partir da vibração da peça, foram gerados pelo equipamento os valores de freqüência e peso. Com esses valores, juntamente com as dimensões das peças e com o valor do vão empregado, foi possível determinar a partir da eq. 2 o valor do módulo de elasticidade dinâmico $\left(\mathrm{E}_{\mathrm{d}, \mathrm{vt}}\right)$.

$$
\mathrm{E}_{\mathrm{d}, \mathrm{vt}}=\frac{\mathrm{fr}^{2} x W x \mathrm{~L}^{3}}{2,46 x I x g}
$$

onde:

fr é a freqüência de ressonância $(\mathrm{Hz})$;

W é a massa da viga $(\mathrm{kg})$;

L é o vão da peça (m);

I é o momento de inércia da seção transversal $\left(\mathrm{cm}^{4}\right)$; e

g é a aceleração da gravidade $\left(9,8 \mathrm{~m} / \mathrm{s}^{2}\right)$.

Simultaneamente aos ensaios de ultra-som e vibração transversal, foram realizados ensaios de flexão estática (MOE) para a obtenção de correlações entre os valores do módulo de elasticidade registrados. As peças foram dispostas na posição de menor inércia e foram submetidas a um carregamento na região central, sendo realizadas seis leituras de deslocamento. O MOE à flexão em relação ao eixo de menor inércia foi determinado utilizando-se a eq. 3 .

$$
\text { MOE }=\frac{\Delta F x L^{3}}{48 x \Delta v x I}
$$

onde:

MOE é o módulo de elasticidade (MPa);

$\Delta \mathrm{F}=(\mathrm{F} 2-\mathrm{F} 1)$ é o incremento de carga $(\mathrm{N})$;

L é o vão da prancha (mm);

$\Delta \mathrm{v}=\left(\mathrm{v}_{2}-\mathrm{v}_{1}\right)$ é o incremento de deslocamento $(\mathrm{mm}) ; \mathrm{e}$

I é o momento de inércia da seção transversal $\left(\mathrm{mm}^{4}\right)$

A Figura 4 ilustra o ensaio de flexão estática.

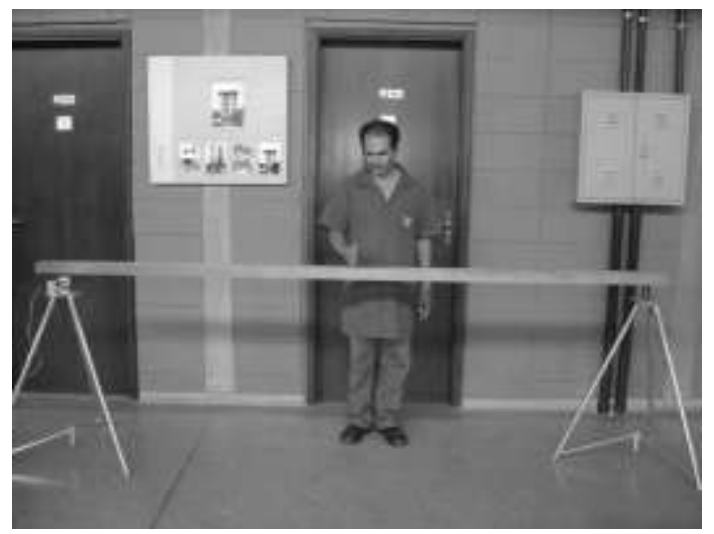

Figura 3 - Ensaio de vibração transversal

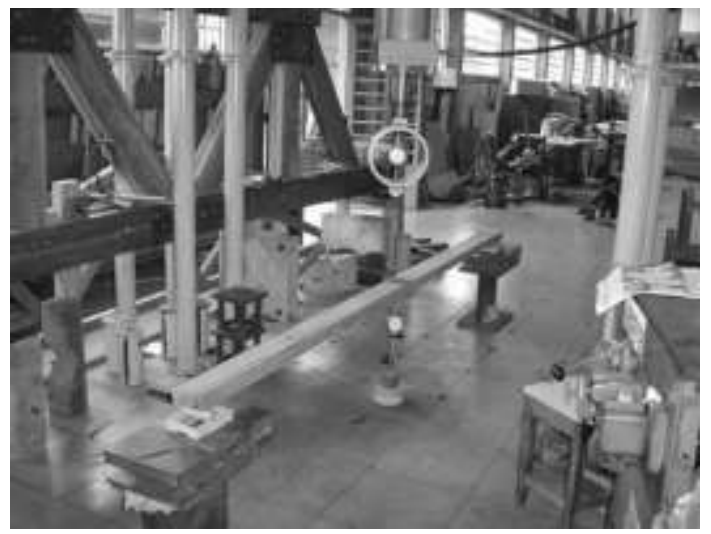

Figura 4 - Ensaio de flexão estática 
Para a determinação da imagem tomográfica do disco de madeira, foi empregado o equipamento de ondas de tensão FAKOPP 2D, o qual pode ser visualizado na Figura 5. O equipamento apresenta oito canais de emissão e recebimento da onda. Ao aplicar um impacto em um dos sensores, o equipamento fornece tempos de propagação da onda no interior do material.

Para a realização do ensaio, os oito transdutores foram fixados no disco de madeira com o auxílio de um martelo, em 8 pontos demarcados, de forma a englobar todo o contorno do disco (Figura 6).

A onda foi emitida no material por meio da aplicação de suaves pancadas com um martelo, conforme ilustrado na Figura 7. O impacto foi dado em cada um dos oito transdutores posicionados no disco. $\mathrm{O}$ equipamento forneceu os tempos de propagação da onda no material, o qual possibilitou, a partir dos valores de distância, a obtenção dos valores de velocidade da onda.

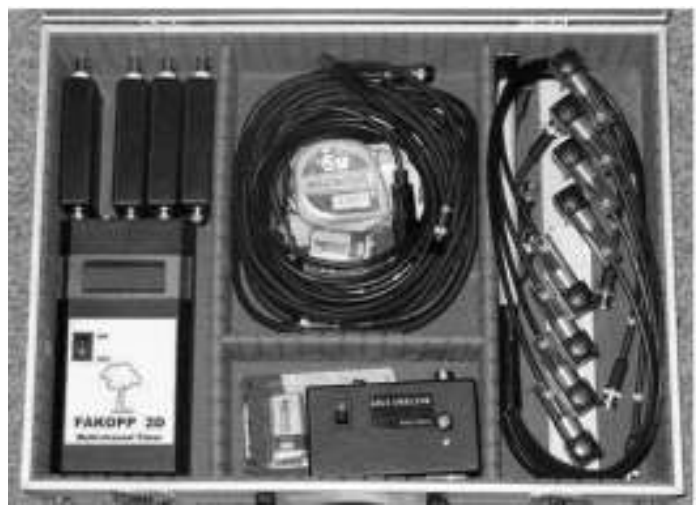

Figura 5 - Equipamento de onda de tensão FAKOPP

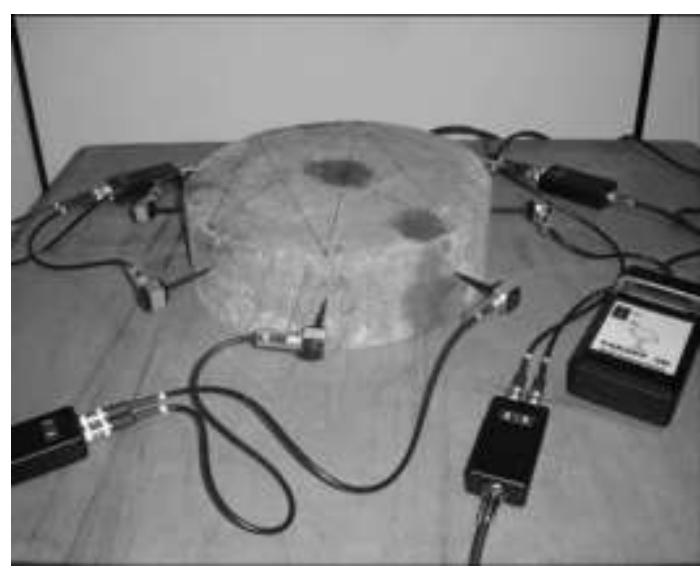

Figura 6 - Ensaio de propagação sonora no disco de madeira

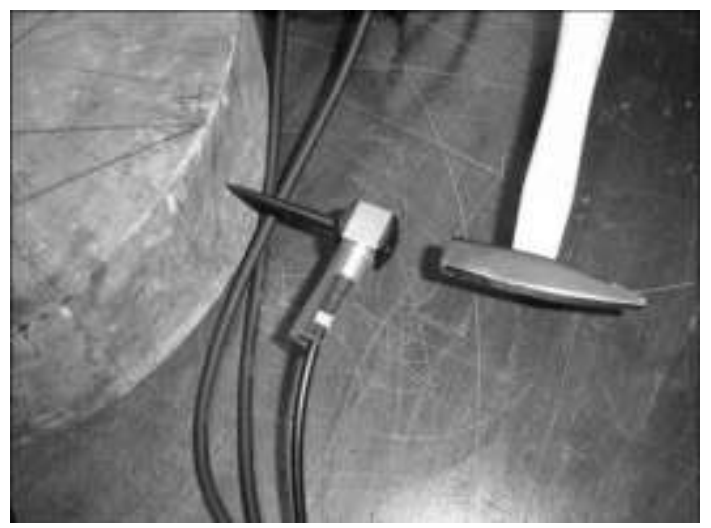

Figura 7 - Aplicação da onda sonora 
Em seguida, foi realizado um furo com $6 \mathrm{~cm}$ de diâmetro no disco de madeira, a fim de se verificar a sensibilidade do equipamento em relação à presença de defeitos na peça (Figura 8).

Para essa nova situação, foi realizado o mesmo procedimento empregado para o disco sem o furo, para a obtenção dos tempos de propagação da onda no material e valores de velocidade (Figura 9), sendo os sensores mantidos na posição inicial.

A partir dos resultados obtidos, foi possível a determinação da imagem tomográfica para o disco com o furo, a qual foi obtida a partir dos valores gerados entre os pontos de transmissão e recepção.

\section{Resultados e discussões}

Neste item estão analisados os resultados obtidos pela realização dos ensaios com as peças estruturais da espécie Eucalyptus citriodora, utilizando-se as técnicas de ultra-som e vibração transversal, os quais foram comparados com os resultados obtidos no ensaio de flexão estática para verificação da acuracidade dessas técnicas nãodestrutivas. Em seguida são feitas considerações sobre a inclusão das técnicas de ultra-som e vibração transversal na NBR 7190:1997. Por fim, estão apresentados e analisados os resultados obtidos para o disco de Eucalyptus citriodora, avaliado pela técnica de ondas de tensão para a obtenção da imagem tomográfica.

\section{Teste de normalidade para os dados obtidos de módulo de elasticidade (estático e dinâmico)}

Para as 30 peças estruturais da espécie em estudo, aplicou-se para os dados obtidos de módulo de elasticidade (estático e dinâmico) o método de Kolmogorov-Smirnov, para verificar se eles apresentavam uma distribuição normal, permitindo, assim, validar estatisticamente as relações obtidas.

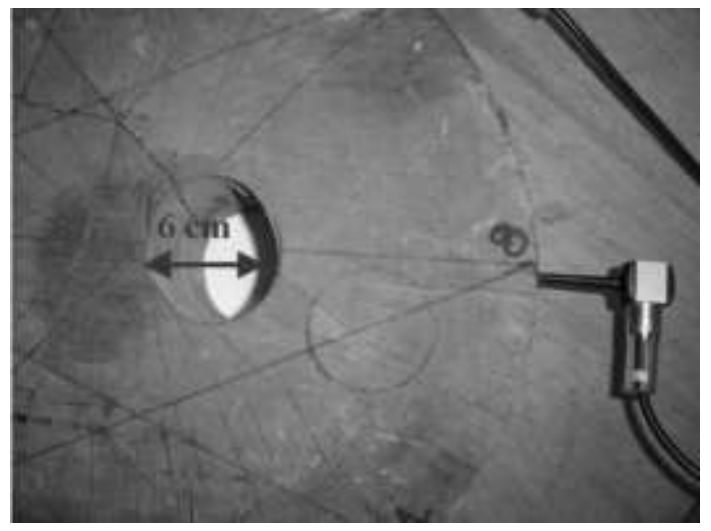

Figura 8 - Disco de madeira com um furo de $6 \mathrm{~cm}$

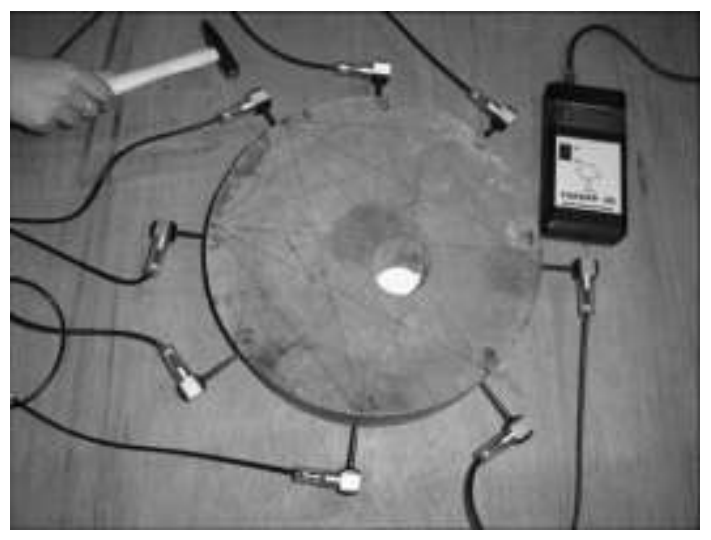

Figura 9 - Propagação da onda sonora no disco perfurado 
O teste consiste em comparar a variável de teste (D), que corresponde à maior diferença observada entre a função de distribuição acumulada do modelo e da amostra, com o valor de D crítico tabelado. A seguir são apresentados os resultados obtidos para a técnica de ultra-som, vibração transversal e flexão estática, para a espécie em estudo.

Para os dados obtidos com a técnica de ultra-som, verifica-se, pelo Figura 10, que o valor obtido para a variável de teste $\mathrm{D}$ corresponde a 0,168 . Para 30 vigas $(\mathrm{n}=30)$, o valor crítico para o nível $\alpha=5 \%$ de significância é 0,242 . Nota-se que 0,168 é inferior a $0,242, \log$ o se pode aceitar a hipótese de normalidade.

Para os dados obtidos com a técnica de vibração transversal, verifica-se, pelo Figura 11, que o valor obtido para a variável de teste $\mathrm{D}$ corresponde a 0,173 . Para 30 vigas $(n=30)$, o valor crítico para o nível $\alpha=5 \%$ de significância é 0,242 . Nota-se que 0,173 é inferior a $0,242, \log$ o se pode aceitar a hipótese de normalidade.

Para os dados obtidos com a flexão estática, verifica-se, pela Figura 12, que o valor obtido para a variável de teste $\mathrm{D}$ corresponde a 0,168 . Para 30 vigas $(\mathrm{n}=30)$, o valor crítico para o nível $\alpha=5 \%$ de significância é 0,242 . Nota-se que 0,168 é inferior a $0,242, \log$ o se pode aceitar a hipótese de normalidade.

Assim, nota-se que ocorre distribuição normal para os dados obtidos em cada uma das técnicas empregadas, sendo possível validar estatisticamente as relações obtidas ao nível de significância de $5 \%$.

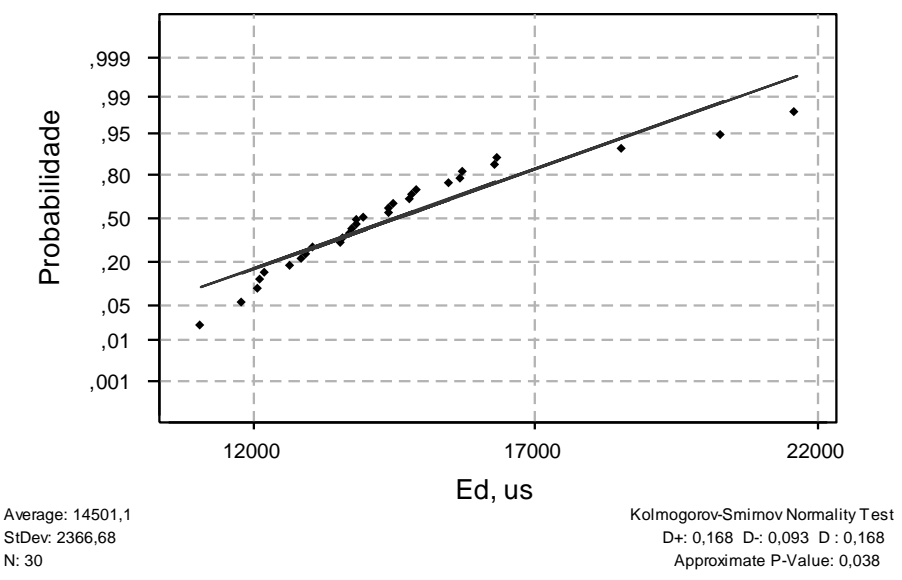

Figura 10 - Figura do teste de Kolmogorov-Smirnov para a espécie Eucalyptus citriodora - ultra-som

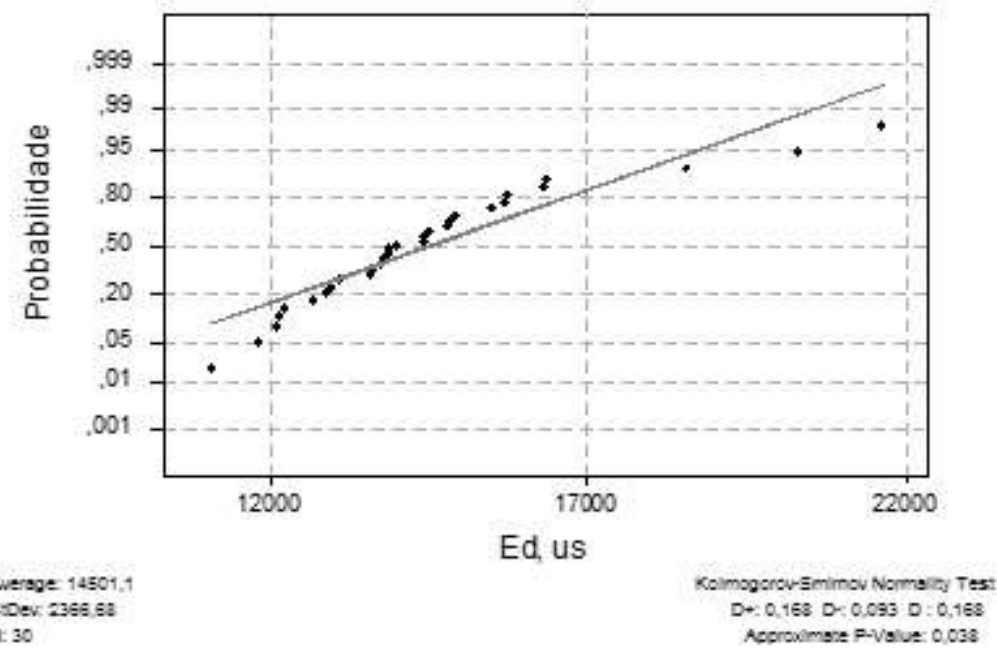

Figura 11 - Figura do teste de Kolmogorov-Smirnov para a espécie Eucalyptus citriodora - vibração transversal 


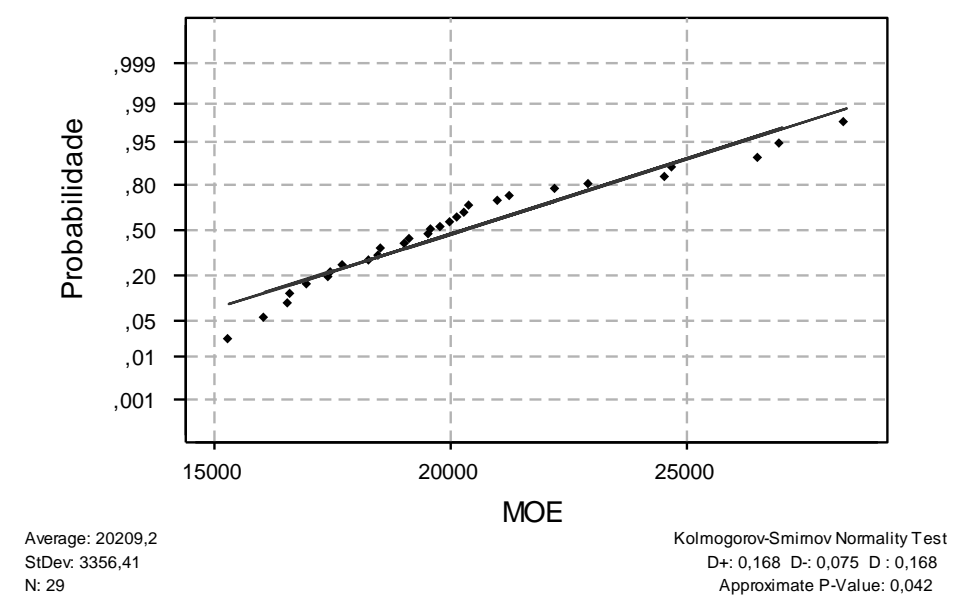

Figura 12 - Figura do teste de Kolmogorov-Smirnov para a espécie Eucalyptus citriodora - flexão estática

\section{Correlação entre o módulo de elasticidade longitudinal e o módulo de elasticidade dinâmico obtido com a técnica de ultra-som}

Na Tabela 1 estão apresentados os valores referentes à densidade, à velocidade ultra-sônica na direção longitudinal, ao módulo de elasticidade dinâmico obtido com a técnica de ultra-som e também ao módulo de elasticidade dinâmico obtido com a técnica de vibração transversal, para as 30 peças estruturais em estudo.

Com os pares de valores dos módulos de elasticidade dinâmico e longitudinal, obtidos para cada uma das peças, realizou-se a regressão linear, apresentada no Figura 13.

$\mathrm{O}$ coeficiente de determinação $\mathrm{R} 2=0,76$ obtido para a espécie em estudo é significativo, compatível com os valores usualmente encontrados na literatura, o que permite afirmar que o método não-destrutivo por meio de ultrasom pode ser utilizado para avaliar as propriedades mecânicas das espécies em questão com dimensões estruturais.

Diversas pesquisas nacionais e internacionais demonstram a viabilidade do método de ultra-som, por meio de correlações entre os módulos de elasticidade obtidos em ensaios destrutivos (compressão paralela às fibras e flexão estática) e em ensaios não-destrutivos com emprego da técnica de ultra-som. Os valores encontrados na literatura indicam valores de coeficiente de determinação $\left(\mathrm{R}^{2}\right)$ entre 0,57 e 0,89 (BARTHOLOMEU，2001; NOGUEIRA，2003;
OLIVEIRA，2005; ROSS; PELLERIN， 1991 1994).

$\mathrm{O}$ coeficiente de determinação $\mathrm{R} 2=0,76$ obtido para a espécie em estudo é significativo, compatível com os valores usualmente encontrados na literatura, o que permite afirmar que o método não-destrutivo por meio de ultrasom pode ser utilizado para avaliar as propriedades mecânicas das espécies em questão com dimensões estruturais.

Diversas pesquisas nacionais e internacionais demonstram a viabilidade do método de ultra-som, por meio de correlações entre os módulos de elasticidade obtidos em ensaios destrutivos (compressão paralela às fibras e flexão estática) e em ensaios não-destrutivos com emprego da técnica de ultra-som. Os valores encontrados na literatura indicam valores de coeficiente de determinação $\left(\mathrm{R}^{2}\right)$ entre 0,57 e 0,89 (BARTHOLOMEU， 2001; NOGUEIRA, 2003; OLIVEIRA, 2005; ROSS; PELLERIN, 1991, 1994).

\section{Correlação entre o módulo de elasticidade longitudinal e o módulo de elasticidade dinâmico obtido com a técnica de vibração transversal}

Na Tabela 2 estão apresentados os valores referentes ao módulo de elasticidade dinâmico obtido com a técnica de vibração transversal e também ao módulo de elasticidade longitudinal obtido para as 30 peças estruturais em estudo. 


\begin{tabular}{c|c|c|c|c|c|c}
\hline \multirow{2}{*}{ Espécie } & & $\begin{array}{c}\rho_{\text {ap, 12\% }} \\
\left(\mathbf{k g} / \mathbf{m}^{3}\right)\end{array}$ & $\mathbf{V}_{\mathbf{L L}}(\mathbf{m} / \mathbf{s})$ & $\begin{array}{c}\mathbf{E}_{\mathbf{d}, \mathbf{u s}} \\
(\mathbf{M P a})\end{array}$ & $\begin{array}{c}\mathbf{M O E} \\
(\mathbf{M P a})\end{array}$ & $\begin{array}{c}\mathbf{E}_{\mathbf{d}, \mathbf{v t}} \\
(\mathbf{M P a})\end{array}$ \\
\hline \multirow{3}{*}{$\begin{array}{c}\text { Eucalyptus } \\
\text { citriodora }\end{array}$} & Valor máximo & 815,8 & 5149,0 & 21625,0 & 28332,0 & 22534 \\
\cline { 2 - 7 } & Valor mínimo & 509,9 & 3804,0 & 11041,0 & 15294,0 & 11267 \\
\cline { 2 - 7 } & Média & 755,1 & 4379,0 & 14501,0 & 20209,0 & 14975 \\
\cline { 2 - 7 } & $\mathbf{C V}(\%)$ & 8,6 & 7,9 & 16,3 & 16,3 & 17,9 \\
\hline
\end{tabular}

Tabela 1 - Valores máximos, mínimos e médios e coeficientes de variação

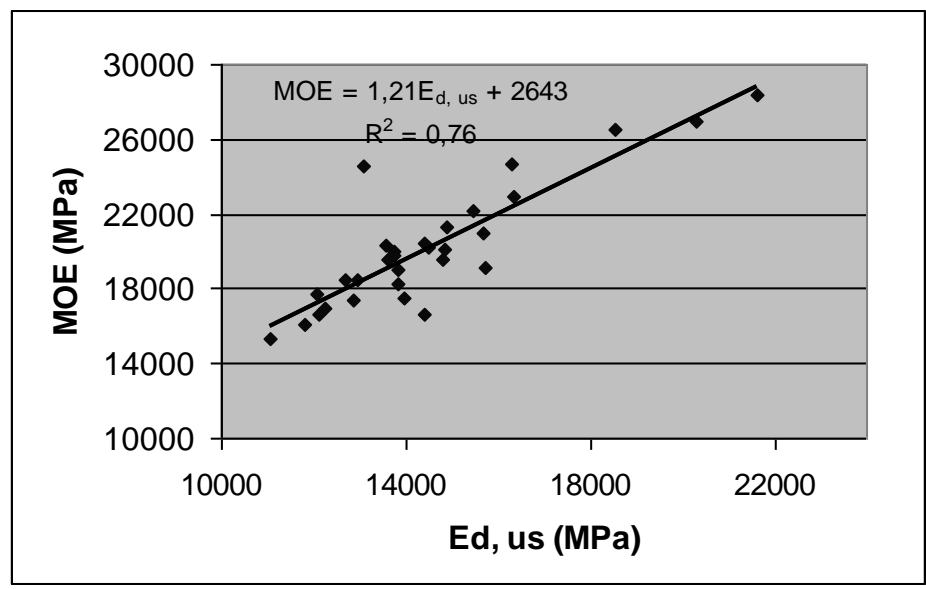

Figura 13 - MOE versus Ed, us - Eucalyptus citriodora

\begin{tabular}{c|c|c|c}
\hline \multirow{2}{*}{ Espécie } & & $\mathbf{E}_{\mathbf{d}, \mathbf{v t}}(\mathbf{M P a})$ & $\begin{array}{c}\text { MOE } \\
(\mathbf{M P a})\end{array}$ \\
\hline \multirow{3}{*}{ Eucalyptus citriodora } & Valor máximo & 22534 & 28332 \\
\cline { 2 - 4 } & Valor mínimo & 11267 & 15294 \\
\cline { 2 - 4 } & Média & 14975 & 20209 \\
\cline { 2 - 4 } & CV (\%) & 17,9 & 16,3 \\
\hline
\end{tabular}

Tabela 2 - Valores máximos, mínimos e médios de módulo de elasticidade dinâmico e longitudinal e coeficientes de variação

Ccom os pares de valores dos módulos de elasticidade dinâmico e longitudinal, obtidos para cada uma das peças, realizou-se a regressão linear, apresentada no Figura 14.

$\mathrm{O}$ valor de coeficiente de determinação $\mathrm{R}^{2}=0,80$ obtido para a espécie em estudo é compatível com os usualmente encontrados na literatura, sendo considerado satisfatório, o que mostra a validade da técnica de vibração transversal na avaliação do módulo de elasticidade.

Diversas pesquisas têm demonstrado a viabilidade do método de vibração transversal na avaliação do módulo de elasticidade da madeira, por meio de correlações entre os módulos de elasticidade obtidos em ensaios destrutivos e em ensaios não- destrutivos com o emprego da técnica de vibração transversal. Os valores encontrados na literatura indicam valores de coeficiente de determinação $\left(\mathrm{R}^{2}\right)$ entre 0,53 e 0,99 (BALLARIN; TARGA; PALMA, 2002; BARTHOLOMEU et al., 2004; BURDZIK; NKWERA, 2002; CALIL JÚNIOR; MINÁ, 2003; CARREIRA et al., 2004; ROSS; PELLERIN, 1991). Os resultados obtidos nesta pesquisa com a aplicação das técnicas nãodestrutivas de ultra-som e de vibração transversal validam e ratificam o emprego dessas técnicas dentro da confiabilidade exigida na construção civil brasileira. Os parâmetros para balizar a utilização normativa dessas técnicas são discutidos e apresentados no item a seguir. 


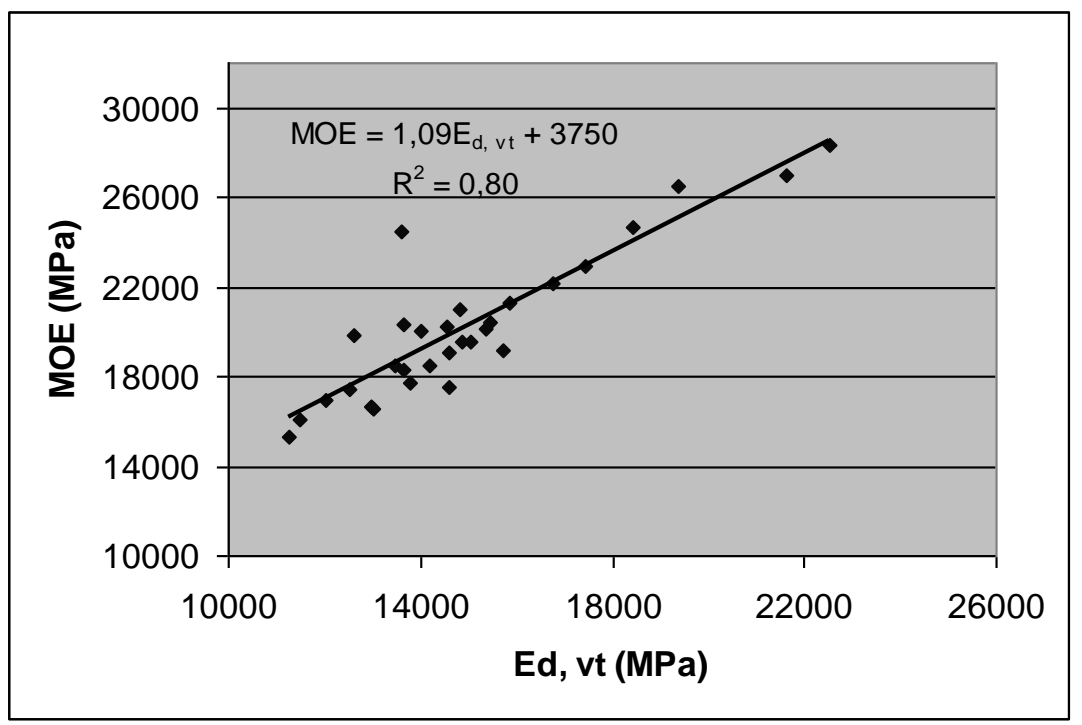

Figura 14 - MOE versus Ed, vt - Eucalyptus citriodora

\section{Considerações a respeito da inclusão das técnicas não-destrutivas de ultra- som e vibração no âmbito da NBR 7190:1997}

Com base nos resultados obtidos em pesquisa desenvolvida por Candian (2007), foi possível sugerir a incorporação dos parâmetros das técnicas não-destrutivas de ultra-som e vibração transversal no âmbito da Norma Brasileira NBR 7190 Projeto de Estruturas de Madeira, no que se refere à classificação mecânica de peças estruturais de madeiras, através da proposição de valores de coeficientes de modificação.

A NBR 7190:1997 especifica em seu conteúdo os coeficientes de modificação, os quais podem ser definidos como números que regulam a estimativa de uma propriedade do material em função de fenômenos que podem ocorrer permanentemente ou durante certo período da vida útil da construção. As especificações da NBR 7190:1997 em relação aos coeficientes de modificação estabelecem três coeficientes parciais de modificação, os quais levam em consideração as condições de carregamento e de umidade, e a qualidade da madeira, denominados respectivamente de kmod,1, kmod,2 e kmod,3.

A especificação de um coeficiente de modificação de acordo com a qualidade da madeira reflete a preocupação com a realização da classificação visual e mecânica, de tal modo que esse material possa ser aplicado com maior confiabilidade e segurança. Ao se comparar a NBR 7190:1997 com algumas normas internacionais, verifica-se que existem especificações distintas para o coeficiente de modificação, e a maior parte das normas utiliza- se de tabelas para classificar a qualidade da madeira nas respectivas classes de resistência, de acordo com a espécie a ser utilizada.

A NBR 7190:1997 estabelece um sistema de classes de resistência que permite o enquadramento de espécies em função de suas propriedades de resistência, estando esse sistema dividido em classes de resistência para as folhosas e em classes de resistência para as coníferas. Tal sistema facilita a especificação da madeira na fase de projeto, permitindo a especificação estrutural da madeira das centenas de espécies nativas e cultivadas no Brasil por meio dos valores das propriedades mecânicas, e não pelo nome científico ou popular da espécie. Todavia, esse sistema de classificação se restringe à fase do projeto. A NBR 7190:1997 não utiliza tabelas de classificação da qualidade da madeira, as quais poderiam auxiliar na fase de recebimento da madeira no canteiro de obras, de modo a homogeneizar os lotes e a aumentar a confiabilidade em relação à classe de resistência especificada em projeto. A inclusão em termos normativos de um coeficiente de modificação (kmod,3) pode auxiliar o meio técnico na fase de classificação visual e mecânica de qualquer espécie dentro das classes de resistência estabelecidas pela referida norma.

Para a determinação dos valores de kmod,3, foram realizados estudos empregando peças com dimensões estruturais de $5 \mathrm{~cm}$ x $11 \mathrm{~cm}$ x $300 \mathrm{~cm}$ das espécies Eucalyptus citriodora, Eucalyptus grandis, Pinus sp e Goupia glabra (CANDIAN, 2007). Além dos ensaios com as técnicas de ultrasom, vibração transversal e flexão estática, foi realizada a classificação visual, classificando-se as peças em classe estrutural especial (SE), classe 
estrutural $\mathrm{N}^{\circ} 1$ (S1), classe estrutural $\mathrm{N}^{\circ} 2$ (S2) e classe estrutural $\mathrm{N}^{\circ} 3$ (S3). A classe SE corresponde à melhor condição de classificação de uma peça, segundo a qual a peça apresenta uma melhor qualidade. A classe S3 corresponde à pior condição de classificação, quando há uma qualidade inferior da peça, com presença de defeitos significativos. As demais classes correspondem a condições de qualidade intermediárias entre a classe de melhor e a de pior qualidade. Com o estudo realizado por Candian (2007) foi possível chegar a valores aplicáveis de kmod,3 para as quatro espécies estudadas, mas, devido ao elevado número de espécies encontradas no Brasil, para a calibração da normalização brasileira, foi necessária a simplificação, a partir dos resultados obtidos para as espécies estudadas, de valores de kmod,3 aplicáveis às folhosas e de valores aplicáveis às coníferas. Entre as coníferas houve ainda a separação entre as coníferas densas e as coníferas não-densas. Com isso foi possível tornar sua aplicação mais simplificada, de fácil aplicação pelo meio técnico. Nas Tabelas 3 a 5 são apresentados os valores de kmod,3 aplicáveis às folhosas, coníferas densas e não-densas, conforme o estudo experimental desenvolvido por Candian (2007).
Os valores dos parâmetros relativos à inclusão das técnicas não-destrutivas apresentados neste artigo têm como finalidade auxiliar no aprimoramento normativo da NBR 7190:1997 no que se refere ao recebimento e à classificação de madeira para uso na construção civil brasileira.

\section{Imagem tomográfica obtida com a utilização da técnica de ondas de tensão}

$\mathrm{Na}$ Tabela 6 estão apresentados os valores obtidos de velocidade de propagação da onda sonora para o disco de madeira sem perfuração, considerando os 8 pontos em que foram instalados os transdutores de transmissão e recepção.

Quando a onda sonora cruza o disco de madeira, por exemplo, entre os sensores 1 e 4 , tem-se a propagação de onda radial. Já quando a onda percorre a distância entre sensores adjacentes, por exemplo, sensores 1 e 2, têm-se ondas tangenciais, as quais possuem menor velocidade de propagação, devido à falta de predominância de elementos anatômicos, como os raios na propagação radial. Essa diferença na velocidade entre ondas radiais e tangenciais pode ser claramente visualizada nos valores apresentados acima.

\begin{tabular}{c|c|c|c}
\hline Classe & $\begin{array}{c}\mathbf{k}_{\text {mod,3 }} \text { (visual } \\
\text { +flexão estática) }\end{array}$ & $\begin{array}{c}\mathbf{k}_{\text {mod,3 }} \text { (visual + vibração } \\
\text { transversal) }\end{array}$ & $\mathbf{k}_{\text {mod,3 }}$ (visual + ultra-som) \\
\hline SE & 1,00 & 0,97 & 0,92 \\
\hline S1 & 0,95 & 0,92 & 0,87 \\
\hline S2 & 0,90 & 0,87 & 0,82 \\
\hline S3 & 0,85 & 0,82 & 0,77 \\
\hline
\end{tabular}

Tabela 3 - Valores de $\mathbf{k}_{\text {mod,3 }}$ aplicáveis às folhosas

\begin{tabular}{c|c|c|c}
\hline Classe & $\begin{array}{c}\mathbf{k}_{\text {mod,3 }} \text { (visual } \\
\text { +flexão estática) }\end{array}$ & $\begin{array}{c}\mathbf{k}_{\text {mod,3 }} \text { (visual + vibração } \\
\text { transversal) }\end{array}$ & $\mathbf{k}_{\text {mod,3 }}$ (visual + ultra-som) \\
\hline SE-D & 1,00 & 0,92 & 0,87 \\
\hline S1-D & 0,95 & 0,87 & 0,82 \\
\hline S2-D & 0,90 & 0,82 & 0,77 \\
\hline S3-D & 0,85 & 0,77 & 0,72 \\
\hline
\end{tabular}

Tabela 4 - Valores de $\mathbf{k}_{\text {mod, } 3}$ para coníferas classificadas como densas (D)

\begin{tabular}{c|c|c|c}
\hline Classe & $\begin{array}{c}\mathbf{k}_{\text {mod,3 }} \text { (visual } \\
\text { +flexão estática) }\end{array}$ & $\begin{array}{c}\mathbf{k}_{\text {mod,3 }} \text { (visual + vibração } \\
\text { transversal) }\end{array}$ & $\mathbf{k}_{\text {mod,3 }}$ (visual + ultra-som) \\
\hline SE-ND & 1,00 & 0,87 & 0,82 \\
\hline S1-ND & 0,95 & 0,82 & 0,77 \\
\hline S2-ND & 0,90 & 0,77 & 0,72 \\
\hline S3-ND & 0,85 & 0,72 & 0,67 \\
\hline
\end{tabular}

Tabela 5 - Valores de $\mathbf{k}_{\text {mod,3 }}$ para coníferas classificadas como não-densas (ND) 


\begin{tabular}{c|c|c|c|c|c|c|c|c}
\hline Sensor & $\mathbf{1}$ & $\mathbf{2}$ & $\mathbf{3}$ & $\mathbf{4}$ & $\mathbf{5}$ & $\mathbf{6}$ & $\mathbf{7}$ & $\mathbf{8}$ \\
\hline $\mathbf{1}$ & - & 1.014 & 1.462 & 1.718 & 1.690 & 1.549 & 1.349 & 951 \\
\hline $\mathbf{2}$ & 966 & - & 1.024 & 1.483 & 1.675 & 1.528 & 1.454 & 1.262 \\
\hline $\mathbf{3}$ & 1.453 & 1.040 & - & 1.169 & 1.500 & 1.458 & 1.522 & 1.505 \\
\hline $\mathbf{4}$ & 1.650 & 1.465 & 1.142 & - & 1.000 & 1.164 & 1.394 & 1.535 \\
\hline $\mathbf{5}$ & 1.651 & 1.666 & 1.481 & 973 & - & 1.053 & 1.462 & 1.633 \\
\hline $\mathbf{6}$ & 1.594 & 1.606 & 1.486 & 1.317 & 1.111 & - & 1.239 & 1.566 \\
\hline $\mathbf{7}$ & 1.349 & 1.514 & 1.570 & 1.486 & 1.515 & 1.230 & - & 1.071 \\
\hline $\mathbf{8}$ & 964 & 1.308 & 1.519 & 1.605 & 1.684 & 1.529 & 1.063 & - \\
\hline
\end{tabular}

Tabela 6 - Valores da velocidade de propagação sonora na peça sem perfuração $(\mathrm{m} / \mathrm{s})$

Na Tabela 7 são apresentados os valores obtidos para a velocidade de propagação da onda sonora entre os sensores para o disco de madeira com o furo de $6 \mathrm{~cm}$.

Comparando-se os valores de velocidade de propagação do disco antes e após o furo (Figura 15), é possível verificar que houve uma diminuição da velocidade nas direções entre os sensores que cruzaram o furo. A direção mais afetada foi entre os sensores 4 e 8 , em função da posição do furo. Nesse caso, a diminuição da velocidade foi de $6,20 \%$ (posição $4-8$ ) e de 9,96\% (posição $8-4$ ). A redução da velocidade entre os sensores 2 e 7 também foi sensível, com os valores de $6,53 \%$ (posição $2-7$ ) e de 10,23\% (posição 7 - 2). A onda sonora se propaga mais rapidamente na madeira sadia em relação à madeira deteriorada. A presença de defeitos na linha entre os transdutores resulta numa diminuição da velocidade de propagação sonora, se comparada com uma velocidade de referência.

Com os valores obtidos de velocidade de propagação da onda no material para o disco perfurado, foi possível a geração automática da imagem tomográfica pelo software Fakopp 2D, que acompanha o equipamento de ondas de tensão utilizado na presente pesquisa. Juntamente com a imagem tomográfica, o software forneceu a porcentagem de área com defeito (furo).

Na Figura 16 é possível visualizar a imagem tomográfica gerada a partir do ensaio com o disco com furo. Pode-se verificar que o furo presente no disco de madeira foi detectado pela imagem tomográfica, representando uma área de defeito correspondente a $4 \%$, valor estimado a partir da imagem esquemática. Ao se comparar com a porcentagem real de defeito, oriunda do furo realizado na peça, verifica-se que o furo corresponde a 3\%, estando próximo ao valor estimado. Ressalta-se que na geração da imagem esquemática foram considerados defeitos em outras regiões do disco, como pode ser verificado próximo aos pontos 1 e 8 da seção apresentada na Figura 16, o que pode explicar a pequena diferença entre os valores reais e os calculados.

\section{Conclusões}

A partir dos resultados obtidos e discussões realizadas foi possível obter as seguintes conclusões:

(a) os valores obtidos de coeficientes de determinação $\left(\mathrm{R}^{2}\right)$ para a espécie em estudo permitiram verificar a acurácia das técnicas de ultra-som e vibração transversal, mostrando que ambas são ferramentas válidas para inferência nãodestrutiva do módulo de elasticidade de peças estruturais de madeira;

(b) o coeficiente de determinação $\left(\mathrm{R}^{2}=0,80\right)$ obtido com a técnica de vibração transversal foi superior ao valor obtido com a técnica de ultrasom $(\mathrm{R} 2=0,76)$. Todavia, nota-se uma grande proximidade entre eles, permitindo verificar que ambas as técnicas podem ser utilizadas no recebimento e na classificação de peças estruturais de madeira, com a vantagem adicional de permitir o exame individual das peças. Ressalta-se que a técnica de vibração transversal apresenta melhores resultados, pois permite uma melhor aderência entre o modelo físico do fenômeno e o correspondente modelo matemático associado;

(c) a imagem tomográfica gerada retratou de forma adequada a presença de defeitos no disco de madeira estudado, com indicação de porcentagem de defeitos muito próximo ao valor real, comprovando ser uma importante ferramenta para a avaliação de defeitos internos em peças de madeira;

(d) quando a onda de tensão cruza o disco de madeira, tem-se a propagação de onda radial. Já quando a onda percorre a distância entre sensores adjacentes, têm-se ondas tangenciais, as quais possuem menor velocidade de propagação, devido à falta de predominância de elementos anatômicos, estando essa diferença de velocidades de acordo com a direção de propagação verificada nos valores obtidos durante a realização dos ensaios;

(e) ao realizar a aplicação de ondas de tensão no disco, com este apresentando o defeito (furo), 
verificou-se que houve uma diminuição da velocidade nas direções entre os sensores que cruzaram o furo. Isso ocorreu porque a onda possui maior velocidade de propagação na madeira sadia em relação à madeira com presença de defeitos (heterogeneidades), havendo uma dispersão da onda quando esta passa por uma região com defeito; e (f) em função da variabilidade intrínseca da madeira, e pelas alterações que as peças podem sofrer ao longo dos anos, tornam-se importante a avaliação e a determinação de suas propriedades mecânicas associadas à obtenção de imagens tomográficas, que permitem uma avaliação mais verossímil da madeira, para que esta possa ser empregada na engenharia com a confiabilidade adequada.

\begin{tabular}{c|c|c|c|c|c|c|c|c}
\hline Sensor & $\mathbf{1}$ & $\mathbf{2}$ & $\mathbf{3}$ & $\mathbf{4}$ & $\mathbf{5}$ & $\mathbf{6}$ & $\mathbf{7}$ & $\mathbf{8}$ \\
\hline $\mathbf{1}$ & - & 1.518 & 1.871 & 1.926 & 1.761 & 1.561 & 1.641 & 1.130 \\
\hline $\mathbf{2}$ & 1.518 & - & 1.644 & 1.950 & 1.865 & 1.495 & 1.359 & 1.453 \\
\hline $\mathbf{3}$ & 1.871 & 1.644 & - & 1.823 & 1.936 & 1.520 & 1.322 & 1.496 \\
\hline $\mathbf{4}$ & 1.926 & 1.950 & 1.823 & - & 1.630 & 1.472 & 1.498 & 1.445 \\
\hline $\mathbf{5}$ & 1.761 & 1.865 & 1.936 & 1.630 & - & 1.774 & 1.851 & 1.671 \\
\hline $\mathbf{6}$ & 1.561 & 1.495 & 1.520 & 1.472 & 1.774 & - & 1.890 & 1.905 \\
\hline $\mathbf{7}$ & 1.641 & 1.359 & 1.322 & 1.498 & 1.851 & 1.890 & - & 1.634 \\
\hline $\mathbf{8}$ & 1.130 & 1.453 & 1.496 & 1.445 & 1.671 & 1.905 & 1.634 & - \\
\hline
\end{tabular}

Tabela 7 - Valores da velocidade de propagação sonora na peça perfurada $(\mathrm{m} / \mathrm{s})$

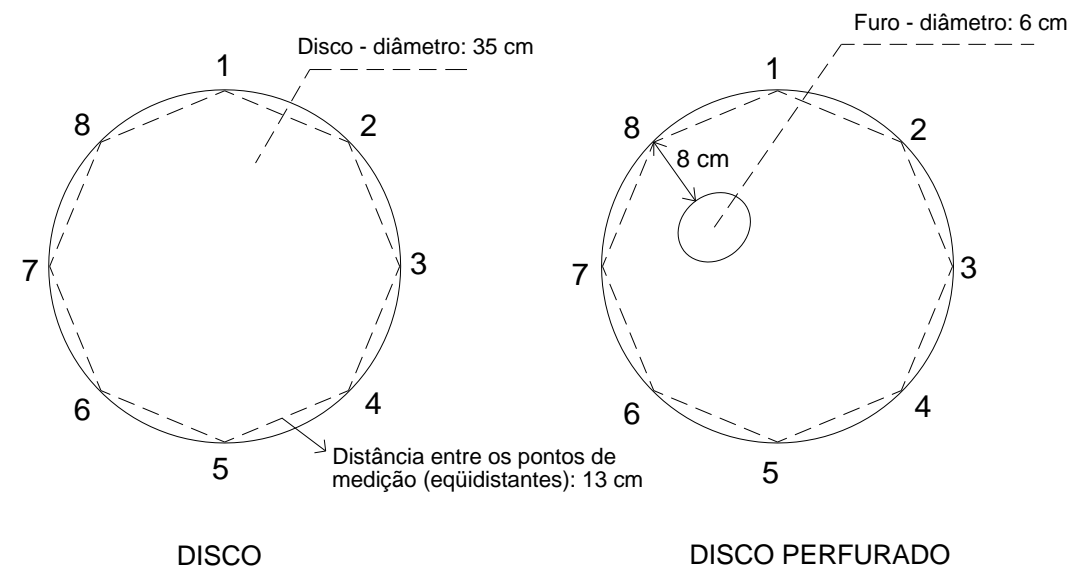

Figura 15 - Esquema do disco: antes e após a realização do furo

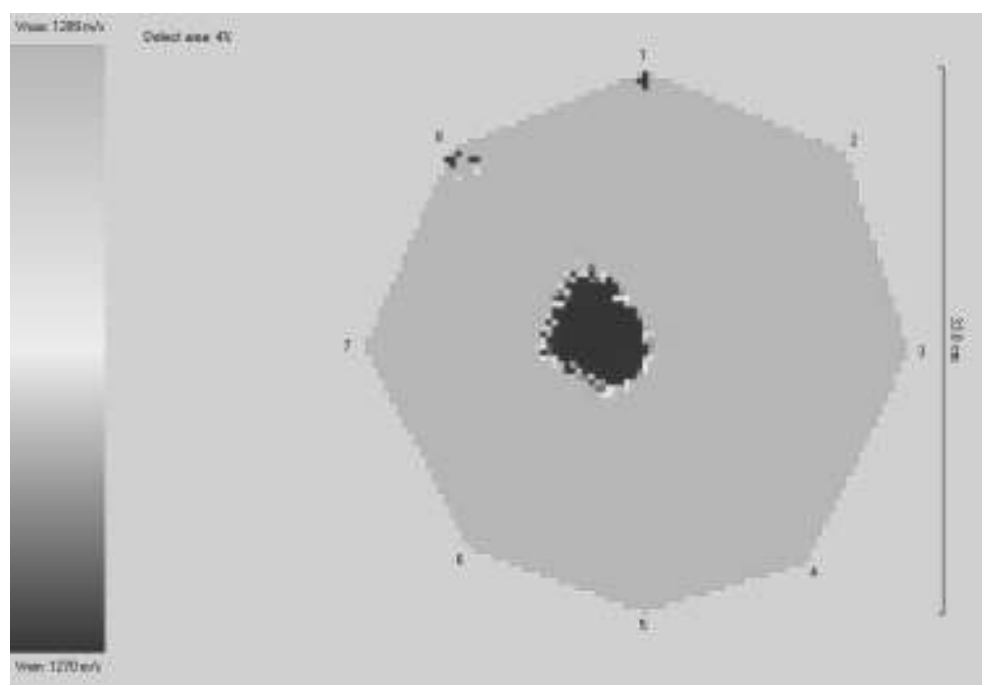

Figura 16 - Imagem tomográfica - disco perfurado 


\section{Referências}

ASSOCIAÇÃO BRASILEIRA DE NORMAS TÉCNICAS. NBR 7190: projeto de estruturas de madeira. Rio de Janeiro, 1997.

ASSOCIAÇÃO BRASILEIRA DE ENSAIOS NÃO-DESTRUTIVOS E INSPEÇÃO. Ensaios Não Destrutivos. Disponível em:

<http://www.abende.org.br/ensaios_nao_destrutivo s.html>. Acesso em: 12 jul. 2006.

BALLARIN, A. W.; TARGA, L. A.; PALMA, H. A. Ensaios Não-Destrutivos de Vibração Transversal na Avaliação do Módulo de Elasticidade de Madeiras de Reflorestamento. In: ENCONTRO BRASILEIRO EM MADEIRAS E EM ESTRUTURAS DE MADEIRA, 8., 2002, Uberlândia. Anais... Uberlândia, 2002.

BARTHOLOMEU, A. Classificação de peças Estruturais de Madeira Através do Ultra-Som. 2001. 73 f. Tese (Doutorado em Ciências e Engenharia de Materiais) - Universidade Estadual de Campinas, Campinas, 2001.

BARTHOLOMEU, A. et al. Metodologia Simplificada para o Ensaio de Vibração Transversal em Vigas Estruturais de Angelim Araroba. In: ENCONTRO BRASILEIRO EM MADEIRAS E EM ESTRUTURAS DE MADEIRA, 9., 2004, Cuiabá. Anais... Cuiabá, 2004.

BUCUR, V. Nondestructive Characterization and Imaging of Wood. Nova York: SpringerVerlag, 2003.

BURDZIK, Walter M. G.; NKWERA, Primus D. Transverse Vibration Tests for Predition of Stiffness and Strength Properties of Full Size Eucalyptus grandis. Forest Products Journal, v. 52, n. 6, p. 63-67, 2002.

CALIL JÚNIOR, C.; MINÁ, A. J. S. Vibração Transversal: um método eficiente para classificação de peças estruturais de madeira. Revista Brasileira de Engenharia Agrícola e Ambiental, v. 7, n. 2, p. 335-338, 2003.

CANDIAN, M. Estudo da Classificação NãoDestrutiva de Peças Serradas de Espécies Cultivadas no Brasil para Uso em Estruturas. 2007. 113 f. Dissertação (Mestrado em Engenharia Civil) - Departamento de Engenharia Civil, Universidade Federal de São Carlos, São Carlos, 2007.
CARREIRA, M. R. et al. Classificação Estrutural de Pinus $s p$ por Vibração Transversal. In: ENCONTRO BRASILEIRO EM MADEIRAS E EM ESTRUTURAS DE MADEIRA, 9., 2004, Cuiabá. Anais... Cuiabá, 2004.

EVANS, R.; KIBBLEWHITE, R. P. Controlling Wood Stiffness in Plantation Softwoods. In: SYMPOSIUM NDT OF WOOD, 12., 2000, University of Western Hungary, Sopron. Proceedings... Sopron, 2000. p. 67- 74.

EVANS, R.; ILIC, J. Rapid Prediction of Wood Stiffness from Microfibril Angle and Density. Forest Products Journal, v. 51, n. 3, p. 53-57, 2001.

FURTADO, E. L. Microrganismos Manchadores da Madeira. Série Técnica IPEF, v. 13, n. 33, p. 91-96, 2000.

GORNIAK, E.; MATOS, J. L. M. Métodos Não Destrutivos para Determinação e Avaliação de Propriedades da Madeira. ENCONTRO BRASILEIRO EM MADEIRA EM ESTRUTURA DE MADEIRA, 7., 2000, São Carlos. Anais... São Carlos, 2000.

HAUFFE, P., MAHLER, G. Evaluation of Internal Log Quality Using X-Ray and Ultrasound. SYMPOSIUM NDT OF WOOD, 12., 2000, Sopron. Proceedings... Sopron: University of Western Hungary, 2000. p. 259-263, 2000.

NOGUEIRA, M. Determinação de Módulos de Elasticidade à Compressão de Madeira de Pinus taeda L. com o Uso de Ultra-Som. 2003. Dissertação (Mestrado em Ciências Agronômicas) - Faculdade de Ciências Agronômicas, Universidade Estadual Paulista, Botucatu, 2003.

MURPHY, J. F. Commentary on Factors Affecting Transverse Vibration Using an Idealized Theoretical Equation. Madison: Forest Products Laboratory, 2000.

NICOLOTTI, G. et al. Application and Comparison of Three Tomographic Techniques for Detection of Decay in Trees. Journal of Arboriculture, v. 29, n. 2, p. 66-78, 2003.

OLIVEIRA, F. G. R. Contribuição ao Estabelecimento de Parâmetros para Ensaios Não-Destrutivos em Madeira Serrada por Meio de Ondas de Ultra-Som. 2005. Tese (Doutorado) - Escola de Engenharia de São Carlos, Universidade de São Paulo, São Carlos, 2005. 
QUOIRIN, N. S. R. Diagnóstico de Amostras de Madeira por Tomografia de Raios-X. 2004. Dissertação (Mestrado em Engenharia Civil) Universidade Federal do Paraná, Curitiba, 2004.

ROSS, R. J.; PELLERIN, R. F. NDE of Green Material with Stress Waves: preliminary results using dimension lumber. Forest Products Journal, v. 41, n. 6, p. 57-59, 1991.

ROSS, R. J.; PELLERIN, R. F. Nondestructive Testing for Assessing Wood Members in Structures: a review. Madison: US Department of Agriculture, 1994.

RUST, S. A New Tomographic Device for the Nondestructive Testing of Trees. In: SYMPOSIUM NDT OF WOOD, 12., 2000, Sopron. Proceedings... Sopron: University of Western Hungary, 2000. p. 233-237.
SANDOZ, J. L. Grading of Construction Timber by Ultrasound. Wood Science and Technology, v. 23, n. 2, p. 95-108, 1989.

\section{Agradecimentos}

À Fundação de Amparo à Pesquisa no Estado de São Paulo (FAPESP), à Coordenação de Aperfeiçoamento de Pessoal de Nível Superior (CAPES), à Universidade Federal de São Carlos (UFSCar), ao Conselho Nacional de Desenvolvimento Científico e Tecnológico (MCT/CNPq) e ao Laboratório de Madeira e Estruturas de Madeira (LaMEM/USP). 